Br J Neurosurg (accepted)

\title{
An unusual cause of sciatica
}

Isabel Tulloch, Marios C. Papadopoulos

Neurosurgery Department, Atkinson Morley Wing, St George's Hospital, London SW19 0QT UK

Corresponding author: isabeltulloch@doctors.org.uk 
A 44-year-old gentleman from Somalia presented with symptoms consistent with right-sided sciatic nerve impingement. He reported that a bullet had ricocheted off a wall and entered his right mid-inguinal region 24 years earlier when he had been living in Somalia during the Civil war. He had made a good initial recovery and in the 18 years that followed he had been completely asymptomatic. Over the last six years, however, he had developed progressively worsening pain and numbness in his right posterior calf and sole when he applied pressure to the posterior aspect of his thigh for example, during sitting.

Interestingly, in the first year after he sustained the injury, he had been able to feel the bullet at the top of his medial thigh approximately $5 \mathrm{~cm}$ below the level of his greater trochanter. Over time, he reported that the bullet had migrated inferiorly. At the time of his presentation, it was palpable within the muscle of his posteromedial thigh, approximately $15 \mathrm{~cm}$ inferior to his greater trochanter.

Figure 1 shows his CT angiogram. This localised the bullet to the adductor magnus, $40 \mathrm{~mm}$ from the sciatic nerve and $5 \mathrm{~mm}$ from the distal tract of the profunda femoris artery. The CT was performed with him lying down. However, his symptoms only occurred when direct pressure was applied to his posterior thigh on sitting. When sitting, due to the body's weight the posterior thigh muscles flatten altering the relative positioning of neurovascular structures in the thigh and in our patient's case may have reduced the distance between the bullet and the sciatic nerve exacerbating the pressure applied to the nerve. His absence of symptoms above the level of the bullet suggested a more proximal cause was less likely. An alternative cause for his symptoms was not identified on the CT. 


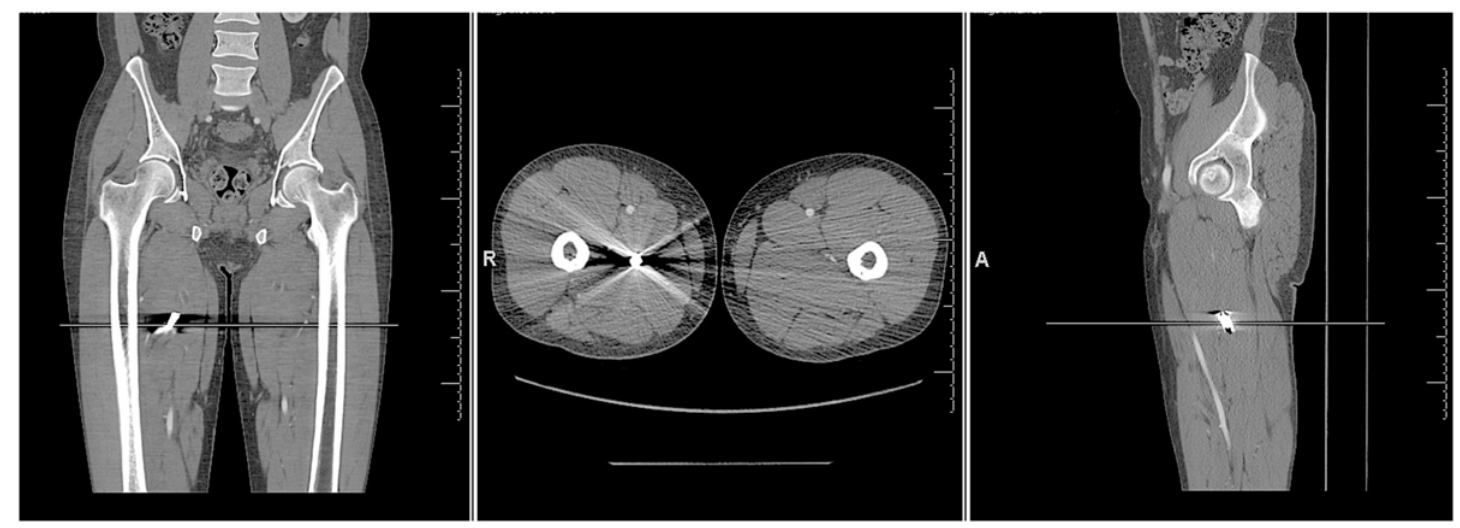

Figure 1. Pre-operative CT angiogram showing the bullet's location in saggital, axial and coronal projections. 


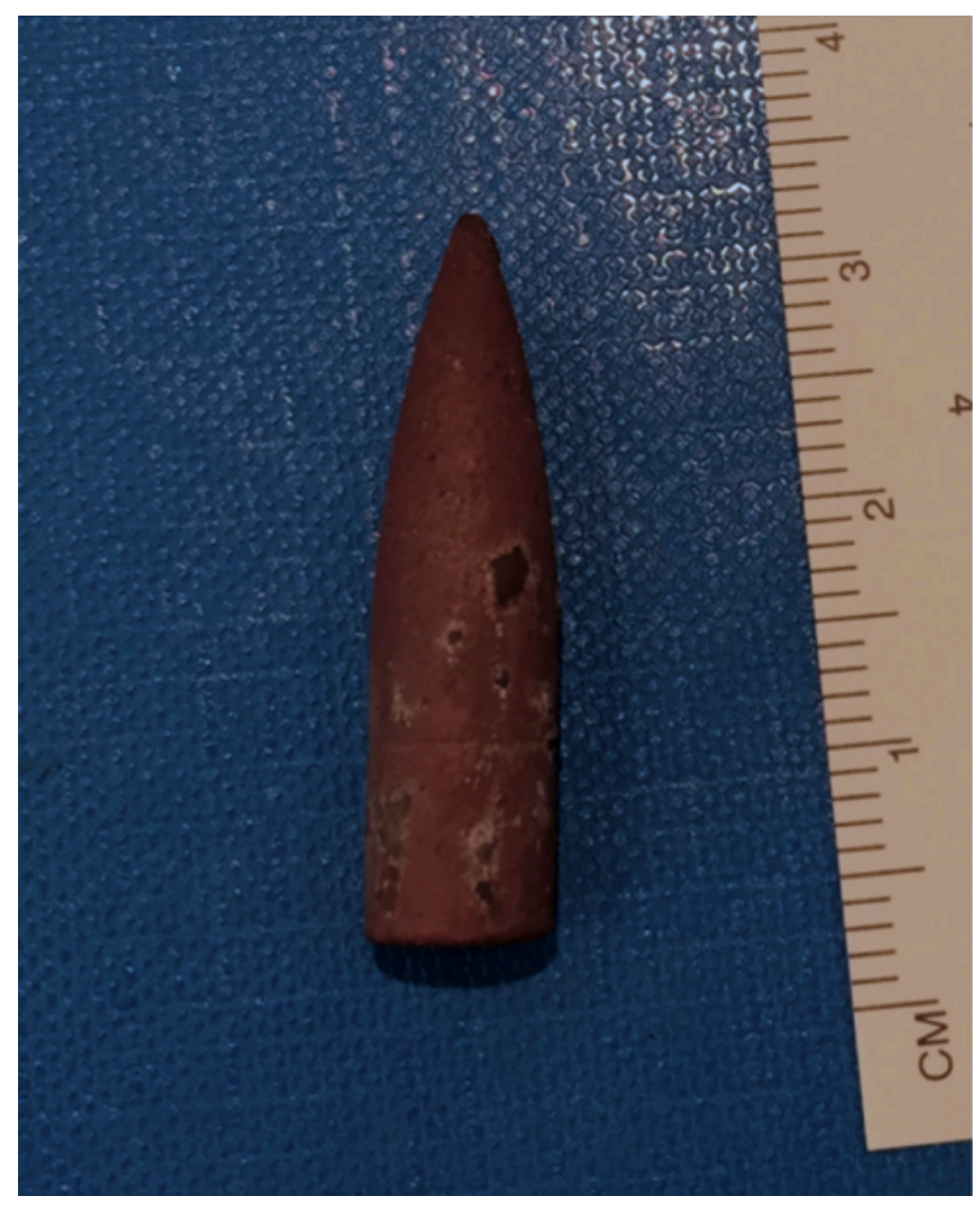

Figure 2. Post-operative image of retrieved bullet. 